\title{
THE APPLICATION OF REMOTE SENSING AND AERO-GEOPHYSICS DATA FUSION ON METALLOGENIC PROGNOSIS IN QIMANTAGE OF EAST KUNLUN MONTAIN AREA
}

\author{
Weijie Jia Hongri Zhang Jian Lin Honglei Zhao \\ China Aero Geophysical Survey \& Remote Sensing Center for Land and Resource, 100083 Xueyuan Road, Haidian \\ District, Beijing \\ sunleaver@gmail.com
}

\section{Commission VII WGVII/6}

KEY WORDS: Qimantage, ASTER, Remote sensing alteration information extraction, High-precision aerogeophysical data, Multiple information fusion model, Geological exploration prospecting

\begin{abstract}
:
Based on west of Qimantage of East Kunlun mountain area, takes advantage of ASTER data, according to the altered mineral spectral characteristics, remote sensing alteration information is extracted. Incorporation the anomaly extraction results with high-precision aero geophysical data processing results, a multiple resource information fusion model is proposed. The fusion model of two totally different type of data which is a special attention in geospatial academia now, which can improve the accuracy of geospatial data application. our fusion result analysis show that it provides information more accurately and sufficiently than separate geospatial data application. The fusion can provide decision-making support for mineral resources prediction.
\end{abstract}

\section{INTRODUCTION}

With the national economy sustained and rapid growth, the demand for mental mineral resource become more and more urgent. Geological exploration technology has been greatly developed now. Along with the constant maturity and application of a large number of high resolution, high precision geology, geophysics, geochemistry and remote sensing prospecting method, abundance data has been provided for geological exploration, metallogenic analysis and prediction (Xing Liu, Guangdao $\mathrm{Hu}, 2003$ ). How to use massive multivariate geospatial data for data analysis and data synthesis, has gradually become the hot spot of geological exploration prospecting technology.

Early in 90s last century, multi-resource geo-spatial information including aero-geophysical, ground geophysical and geochemical exploration and remote sensing had been used to develop a unified format geological image data set and comprehensive analysis and processing based on it, which provides a new potential for mineral exploration interpretation at those time(Dianlin Shi, Yujun Zhang, 1991). But due to the technical ability and data quality, remote sensing information was not as important composition analysis using in comprehensive data model. The basic conception and model building methods of Geosciences data sets had been studied then, and data fusion technology characteristics and their methods which were commonly used in geological data had been discussed(Peigen Dai, etc 2001; Jun Li, Dajun Zhuang, 2001 ). However, there was no application example analysis in metallogenic prediction. Based on research on spatial data characteristics of remote sensing, geophysical and geochemical in his doctoral thesis(Jun Pan,2005), the association rules analysis method for multivariate data fusion methods and visual data mining methods had been researched, which to realize multiple targets information extraction based on geo-spatial data. The key of research was on fusion of remote sensing data and geochemical data yet, the effect of geophysical data was not detail researched.

In our thesis, remote sensing data alteration anomalies information extraction and geophysical data processing have been proceed. The fusion model based on correlation between abnormal information has been studied then. And fusion result has analysis, which provide information more accurate and sufficient than separate result. The fusion can provide decision-making support for mineral resources prediction. 


\section{MULTIPLE INFORMATION FUSION MODEL}

Based on comprehensive analysis research achievement in various of fields at home and abroad, data fusion can be defined as: under a certain criterion of analysis, integration, domination and application, make full using of multi-sensor observation data of different time and space, consistent explanation and description of object can be acquired, the corresponding decision and estimation can be realized in turn, which make the system gets more sufficient information than each other part(Yuanming Gong, etc,2001).

The purpose of metallogenic prognosis based on geological metallogenic regularity in research area, under the guidance of metallogenic theory, using technology of remote sensing, geophysical and geochemical exploration, to predict delineating metallogenic target and evaluate resources. For processing metallogenic prediction based on multiple resource information fusion technology, the spatial relationships and data model unity problem between different data sources should be solved at first time. In expression form, geo-spatial data can be divide into: vector structure data, which point, line, surface elements are used in the data set, and $x, y$ coordinates are used as location identifier, such as geographic data, geological data and some geophysical geochemical exploration data; raster structure data, which uses unit grid of rows and columns as target entity location symbols, and image grey value identity raster data value in the corresponding position, such as geography maps, geology maps and remote sensing images; and other experimental and description data.

The data fusion methods of remote sensing image, aero-geophysical survey data, remote sensing alteration extraction information and aero-geophysical processing data and how to use fusion results in metallogenic prognosis are mainly studied in this paper. Because of current relatively mature data fusion methods mostly used on high resolution and multispectral remote sensing images data fusion, therefore the principle of multi-resources remote sensing images fusion method is using for reference in our research of multiple geo-spatial data fusion.

First of all, ASTER remote sensing image and high-precision aeromagnetic data in study area are processing respectively. The alteration information is extracted using principal component analysis (PCA) method. Mineral alteration component is acquired, and the levels of alteration information are determined in grey level image.
The processing of gridding, reduced to the pole, filter and removed the earth background magnetic field are conducted on original measurement obtained high-precision aeromagnetic data. The color result turn into grayscale image finally. To step the result on the unity of the coordinate system and image registration. The geophysical prospecting and remote sensing extraction information are comprehensive then. Applying interpolation technology to obtain comprehensive results image the same pixel density as the ASTER image.

Because the correlation between ASTER2, ASTER3 and ASTER7 bands is not strong, and they can reflect the different spectrum characteristics of objects, thus the RGB combination of ASTER data adopts ASTER7, ASTER3 and ASTER2 bands(expressed as $A_{R}, A_{G}, A_{B}$ ), to do IHS transform, the transform formula is:

$$
\begin{aligned}
& I=\frac{1}{3}\left(A_{R}+A_{G}+A_{B}\right) \\
& G=\arctan \left[\frac{2 A_{R}-A_{G}-A_{B}}{\sqrt{3}\left(A_{G}-A_{B}\right)}\right]+C \\
& C=0 \text { when } A_{G} \geq A_{B} \text { or } \pi \text { when } A_{G}<A_{B} \\
& S=\frac{\sqrt{6}}{3} \sqrt{A_{R}^{2}+A_{B}{ }^{2}+A_{G}{ }^{2}-A_{R} A_{B}-A_{B} A_{G}-A_{R} A_{G}}
\end{aligned}
$$

The brightness component(I) in hue(H), Saturation(s) and brightness component(I) is replaced by comprehensive results grayscale image. The inverse transformation of RGB to HIS is processing, then the final fused image is acquired( the work flow as shown in figure1)

\section{DATA PROCESSING ANALYSIS}

\subsection{Remote sensing alteration extraction}

In the process of hydrothermal mineralization, a series of change of the composition, structure and construction produced by chemical reaction of wall rock near ores and hydrothermal fluid is called the wall rock alteration. Alteration minerals are the important prospecting marks. Alteration phenomenon can not only indentifies the ground ore body shape and location, but also indicates the existence of underground deposits. Using composition, distribution scope and intensity of the alteration rock, mineral species, occurrence location and enrichment strength can be predicted. Refers to the alteration of remote sensing alteration information is conductive to mineralization occurs within a region of space, the alteration of surrounding rock (belt) reflected on the remote sensing image contains all kinds of background spectrum information, which is integrated 


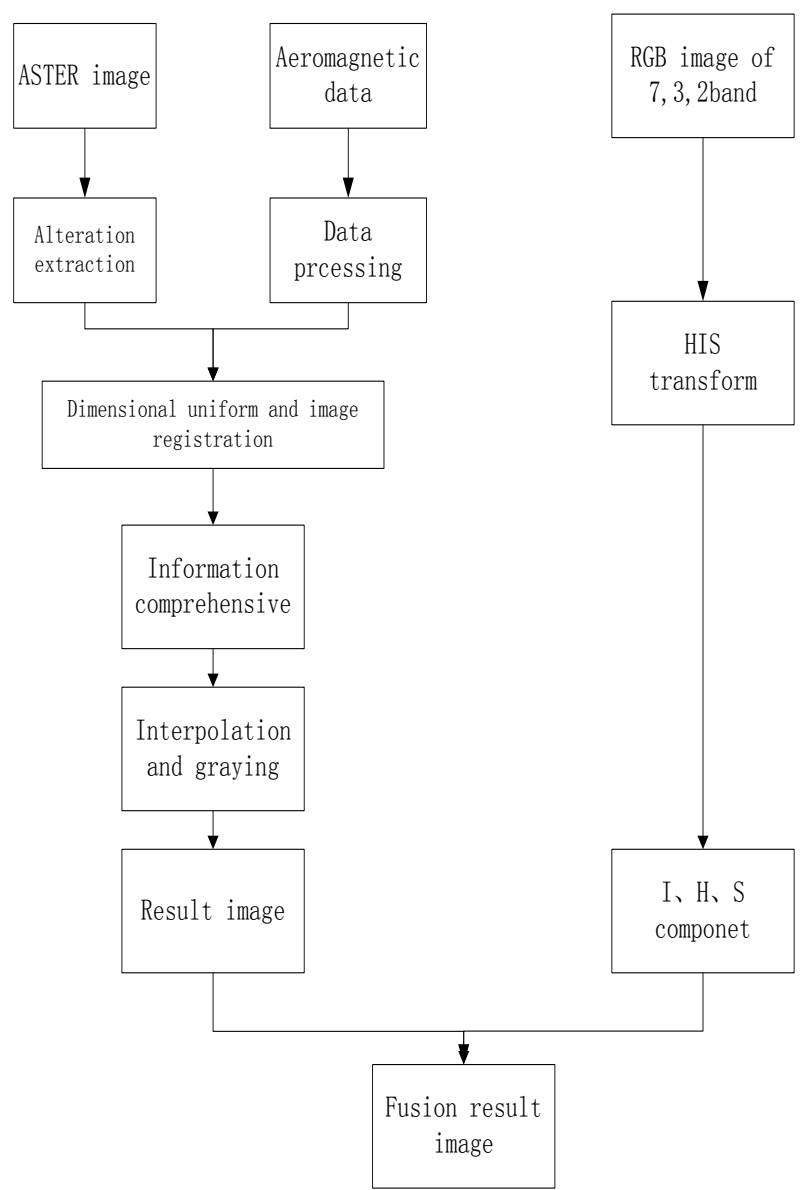

figure1. the flow chart of data fusion

spectral information.

ASTER is multispectral observatory carried by earth observation system (TERRA) satellite launched in December 1999 by NASA. One of main purpose of its development is improving the efficiency of resource exploration. It with high spatial resolution (the resolution of visible- near infrared band is $15 \mathrm{~m})$, there are plenty of spectral channels. In short wavelength infrared, ASTER has six band in alteration spectrum band, which making identification of specific alteration mineral assemblage is easier. There are also five bands in the infrared area, making it to identify the lithology in research possible.

Principal component analysis (PCA) is current widely used mineral alteration information extraction method. The principle of PCA is using linear transformation to decorrelation. After transformation, the information duplication and redundancy are removed between principal component, and the weak alteration information is extracted. According to altered mineral spectrum feature, principal components transformation bands are selected. Analysis size and symbol of load factors of eigenvector after transformation, determine each spectral response contributions of each band to mineralized alteration, the suitable principal components for mineral alteration extraction can be identified. The abnormal size can be graded.

According to the reflective spectrum characteristics of common minerals, refer to "De-interfered Anomalous Principal Component" extraction method( Yujun zhang, etc,2002). Current usually used band combinations are as follows: principal component analysis method of ASTER 1,2,3,4 can be used to extract remote sensing iron anomaly information; principal component analysis method of ASTER1,3,4,5 can be used to extract carbonic acid root remote sensing anomaly information; principal component analysis method of ASTER1,3,4,8 can be used to extract hydroxyl remote sensing anomaly information.

\section{APPLICATION EXAMPLE}

Study area is located in north rim of Qimantage of East Kunlun mountain, southeast margin of the Tarim basin. It belong to Paleozoic island arc belt on tectonic division, which has undergone multiphase and multistage geodynamic evolution, and has complex geological structure, rich in minerals. Formation magmatic rocks of study area is mainly controlled by two major tectonic system, which are ne-trending Arkin fault zone and nww-trending central Kunlun fault zone. The two regional deep faults across the district, constitute a complex tectonic framework, which have obvious effect on regional mineralization control. There are multiple mental points have been found in this area. Major alteration type is given priority to skarnized, there are also include silicification, carbonatization, chloritization and epidotization. As the study area belongs to the alpine region, peak elevation difference is very big, mostly area is uninhabited, geology exploration degree is low. Therefore, the using of method of remote sensing and geophysical prospecting technology can provide early technical support for geological exploration prospecting.

Taking one view of ASTER image on Jun1, 2003 of Qimantage of East Kunlun mountain as example (figure 2). Using reflection data after pretreatment processing and interference removing, the combination of ASTER1,3,4,8 bands have been chosen for PCA transform. Analysis size and symbol of load factors of eigenvector after transformation, determine each spectral response contributions of each band to mineralized alteration, the suitable principal components for mineral alteration extraction can be identified. The greater of 
value of principle component, the greater of the chance of existence of alteration mineral (figure 3).

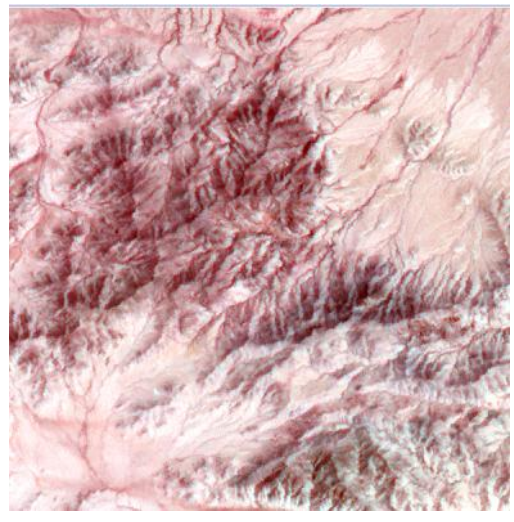

figure2. Remote sensing original image

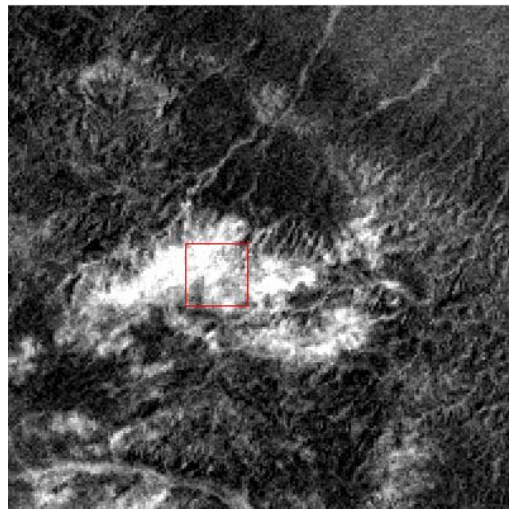

figure3. Remote sensing alteration image

The processing of gridding, reduced to the pole, filter and removed the earth background magnetic field are conducted on original measurement obtained high-precision aeromagnetic data. Aero-magnetic residual contour data is acquired finally. Using the transformation formulas to convert color image to gray image (figure 4). After dimension uniformed and registration, it integrate with mineral alteration information.

After HIS transform of RGB image combine with ASTER 7,3,2 Bands, using integrated grayscale image which including mineral alteration information and aero-magnetic residual data as brightness component, the HIS inverse transform would be done, which acquire finally fusion images (figure.5)

The result fusion image retains the original color and texture information of remote sensing image, which can be undertook topography, lithology, fracture and structure interpretation, at same time contains the mineral alteration information and aero-magnetic contour information, which can infer the mental metallogenic belt and the possible existence of concealed orebody.

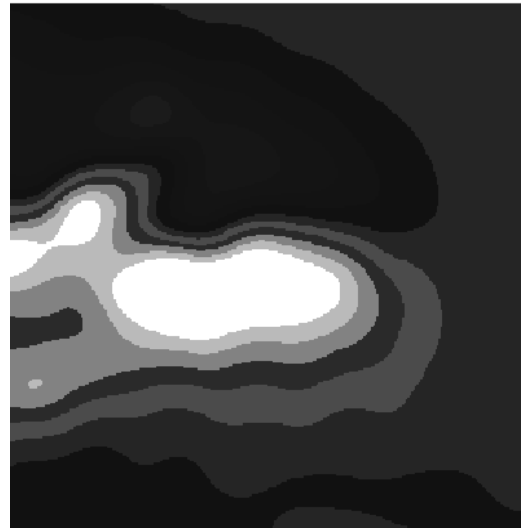

figure.4 Aero-magnetic residual contour

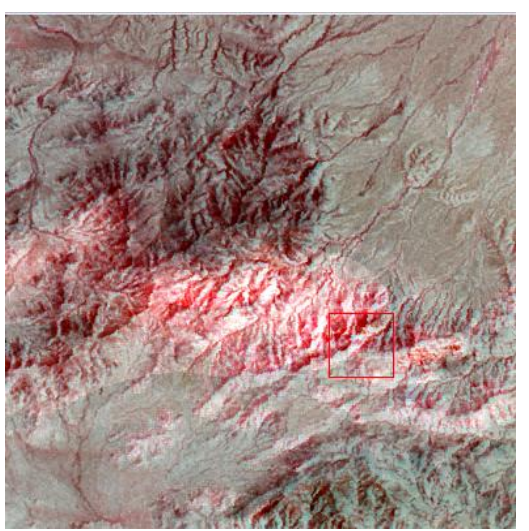

figure.5 fusion image

5. CONCLUSION

As show in experimental results, the fusion of remote sensing and geophysical information can provide reliable discriminate basis for metallogenic rock, fracture, regional tectonic and latent ore, and intuitive reflect the target information in image. However, Because there is large difference between two kinds of data in acquisition principle, imaging methods and expression connotation, the correlation between them still need further research. Putting forward more accurate and intuitive data model and promoting technology practically use will be the following objective of our research.

\section{References}

Dianlin Shi, Yujun Zhang, 1991, Multivariate geological comprehensive images processing method in the application of the mineral resources prediction. The computer application study on geology science, pp. 127-134.

Jun Li, Dafang Zhuang, 2001, Theories and system of geo-spatial data integration. Advances in Earth Science, 20(2), pp. 137-144. 
International Archives of the Photogrammetry, Remote Sensing and Spatial Information Sciences, Volume XL-7/W1, 3rd ISPRS IWIDF 2013, 20 - 22 August 2013, Antu, Jilin Province, PR China

Jun Pan, 2005. Study of data fusion and visual for multisource geo-special data. PhD thesis, University of Jilin, pp. 25-32.

Ta gen Dai, ect, 2001, Geoscientifical data model and research of data fusion technology. Journal of Central South University, 32(3), pp.221-223.

Xing Liu, Guangdao Hu, 2003, Multi-source geo-data fusion and its application in metallogenic prognosis. Acta geoscientia sinica, 24(5), pp. 463-468.

Yuanming Gong, Deyun Xiao, Junjie Wang, 2001, Application of multisensor data fusion techniques in automatic vertical drilling detecting system, 26(5), Earth Science - Journal of China University of Geosciences, pp. 524-528.

Yujun Zhang, Jianmin Yang, Wei Chen, 2002, A study of the method for extraction of alteration anomalies from the ETM (TM) Data and its application. Land resource remote sensing, (4), pp. 47-53.

\section{Acknowledgements}

Acknowledgements of support for the project of "1:50000 Aero-magnetic investigate on Qimantage of East Kunlun Mountain on Xinjiang province ". 